J3eA, Journal sur l'enseignement des sciences et technologies de l'information et des systèmes, Volume 4, Hors-Série 2, 16 (2005)

DOI : http://dx.doi.org/10.1051/bib-j3ea:2005716

(C) EDP Sciences, 2005

\title{
Récepteur hétérodyne pour la bande FM
}

A. Carcy et M. Tournoud

${ }^{1}$ Lycée Joliot Curie

19 bd Joliot Curie

F-34200 Sète, France

2 Lycée J.F. Champollion

avenue de Figuières

BP 43

F-34972 Lattes, France 


\title{
RECEPTEUR HETERODYNE POUR LA BANDE FM
}

\author{
A. Carcy ${ }^{*}$, Lycée Joliot Curie, 19 Bd Joliot Curie, 34200 SETE \\ armelle-carcy@ac-montpellier.fr \\ M. Tournoud, Lycée J.F. Champollion, Av de Figuières, BP 43, 34972 LATTES \\ m.tournoud@wanadoo.fr
}

\begin{abstract}
Résumé : Cet article présente une maquette de récepteur hétérodyne pour la bande $\mathrm{FM}$, conçue pour être utilisée en BTS Electronique. Dans un premier temps, les étudiants étudient le récepteur FM (transposition de fréquence et visualisation des spectres, caractéristiques du démodulateur en quadrature), en utilisant un synthétiseur externe. Puis ils mettent en cuvre le synthétiseur intégré à la maquette (mise en œuvre de l'oscillateur local et du synthétiseur dont les registres sont configurés par PC). Enfin, ils programment un microcontrôleur afin de configurer les registres du synthétiseur par l'intermédiaire d'une liaison SPI. Ces TP permettent aux étudiants d'avoir les outils nécessaires pour réaliser leur propre récepteur pour la bande FM ou des récepteurs à double changement de fréquence pour la bande radioamateur.
\end{abstract}

Mots clé : récepteur hétérodyne, transposition de fréquence, démodulateur en quadrature, logique programmée, liaison SPI

\section{OBJECTIFS :}

Cet article présente une maquette de récepteur hétérodyne pour la bande FM que nous avons réalisée dans le cadre de l'enseignement de la physique appliquée et de l'électronique en BTS électronique. Cette maquette permet d'étudier un récepteur à simple changement de fréquence, avec un démodulateur en quadrature et comporte un synthétiseur de fréquence programmable. Elle a été conçue pour :

- Illustrer une partie du programme (transposition de fréquence, synthétiseur de fréquence, démodulation de fréquence)

- Travailler dans une bande de fréquence utilisée dans la vie courante: la bande FM permet aux étudiants d'écouter la radio, ce qui les motive

- Réaliser des maquettes peu onéreuses et facilement reproductibles afin que plusieurs étudiants puissent travailler en même temps

- Travailler avec les mêmes composants en physique appliquée et en électronique et réaliser un projet commun et complet (électronique analogique et numérique)

- Donner la possibilité aux étudiants de réaliser leur propre récepteur

Les étudiants travaillent sur cette maquette pendant :

- $4 \mathrm{H}$ en TP de physique appliquée pour étudier la transposition de fréquence et le démodulateur de fréquence, avec une préparation faite avant le TP et inspirée d'un sujet de physique appliquée de BTS [1]

- $12 \mathrm{H}$ en mini projet d'électronique avec $4 \mathrm{H}$ consacrées au synthétiseur de fréquence, programmé pour cette partie par $\mathrm{PC}$, et $8 \mathrm{H}$ consacrées à la programmation du synthétiseur par microcontrôleur.

\section{PRESENTATION DE LA MAQUETTE :}

La maquette (fig 1) comporte trois modules :

- Un module récepteur construit autour

- du MC3362 [2] de chez Motorola qui est un récepteur à double changement de fréquence (un seul est utilisé ici) avec un démodulateur à quadrature et un oscillateur local commandé en tension

- du MC145170 [3] de chez Motorola qui est un synthétiseur de fréquence comportant deux diviseurs de fréquence, un comparateur de phase trois états et un oscillateur à quartz. La programmation des registres du synthétiseur se fait par l'intermédiaire de trois fils au format SPI. Sur ce module, des fiches SMB et $2 \mathrm{~mm}$ permettent de connecter un synthétiseur externe et de visualiser les spectres

- Un module microcontrôleur qui comporte un affichage de type LCD, deux touches "plus" et "moins", et un microcontrôleur 89S53 de chez Atmel [4] avec une mémoire de code de type flash, programmé in situ en langage évolué

- Un module haut-parleur (non représenté sur la fig 1) qui comporte un amplificateur basse fréquence et un haut parleur.

\section{ETUDE DU RECEPTEUR FM :}

L'étude du récepteur FM (transposition de fréquence et démodulateur en quadrature) se fait en TP de physique appliquée $(4 \mathrm{H})$.

Ce TP illustre les cours sur la transposition de fréquence et la démodulation de fréquence et doit permettre aux étudiants de comprendre et mettre en œuvre un récepteur hétérodyne.

\subsection{Transposition de fréquence :}

Le but est de visualiser les spectres, avec un analyseur de spectre, dans le cas de réceptions infradyne et supradyne, pour différentes fréquences de l'oscillateur local et de voir le rôle du filtre à fréquence intermédiaire (ici, FI = 10,7 MHz). Pour ces manipulations, le circuit LC de l'oscillateur local interne au MC3362 est déconnecté et un synthétiseur, de fréquence $\mathrm{f}_{\mathrm{OL}}$ (niveau de sortie : -10 $\mathrm{dBm}$ ) est connecté sur "l'entrée synthétiseur". Les spectres sont relevés sur les sorties "spectre avant FI" et "spectre après FI". 


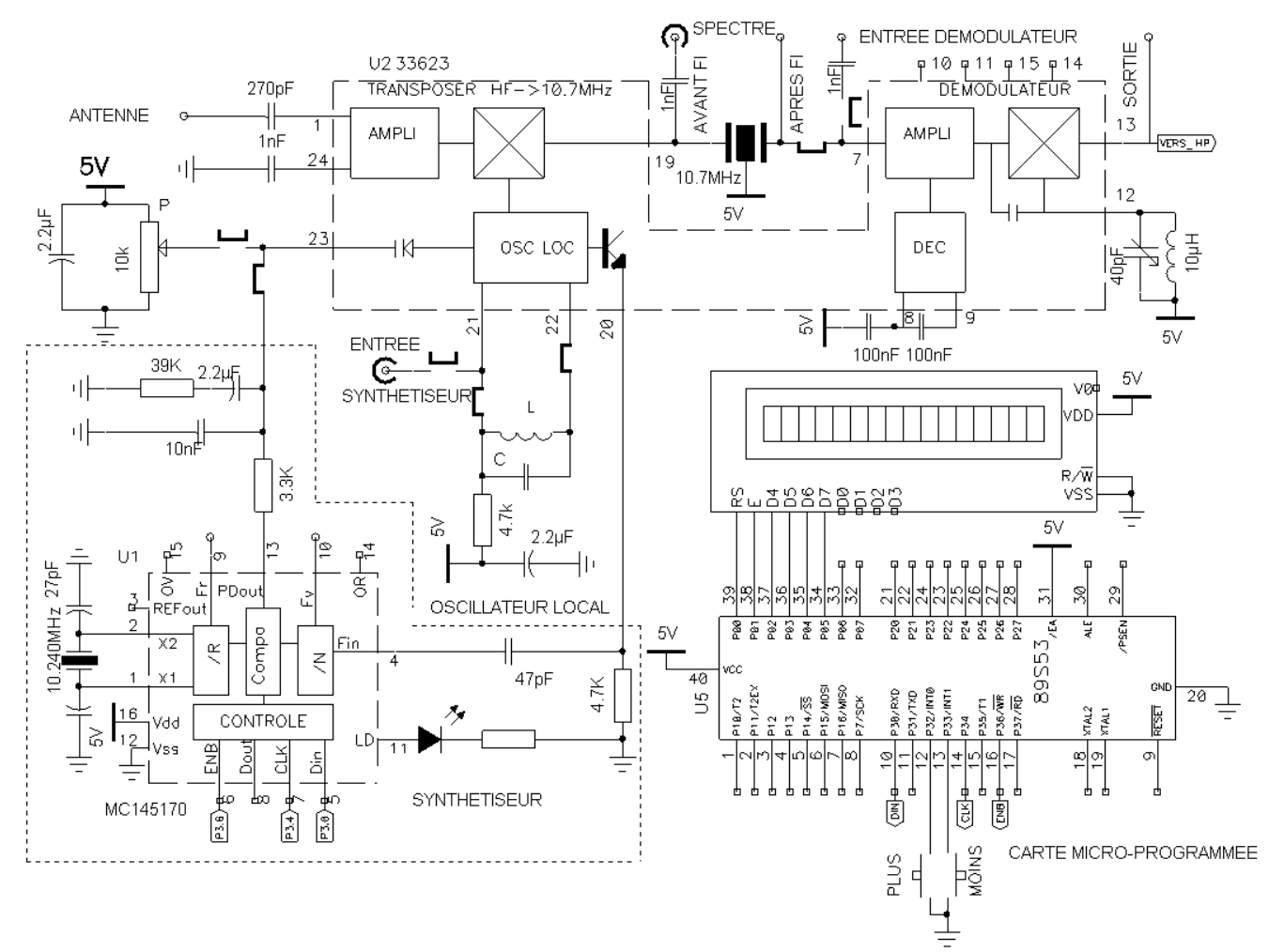

fig 1 : schéma structurel simplifié de la maquette.

Pendant cette première partie, les étudiants doivent :

- Relever le spectre de la bande FM (synthétiseur éteint) (fig 2 a)

- Relever le spectre autour de FI dans le cas d'une réception infradyne (fig $2 \mathrm{~b}$ )

- Relever le spectre autour de FI dans le cas d'une réception supradyne (fig $2 \mathrm{c}$ )

- Observer le rôle du filtre FI (fig 2 d)

Le principal intérêt pédagogique de cette partie est qu'en faisant varier la fréquence du synthétiseur, les étudiants visualisent la translation du spectre à FI.

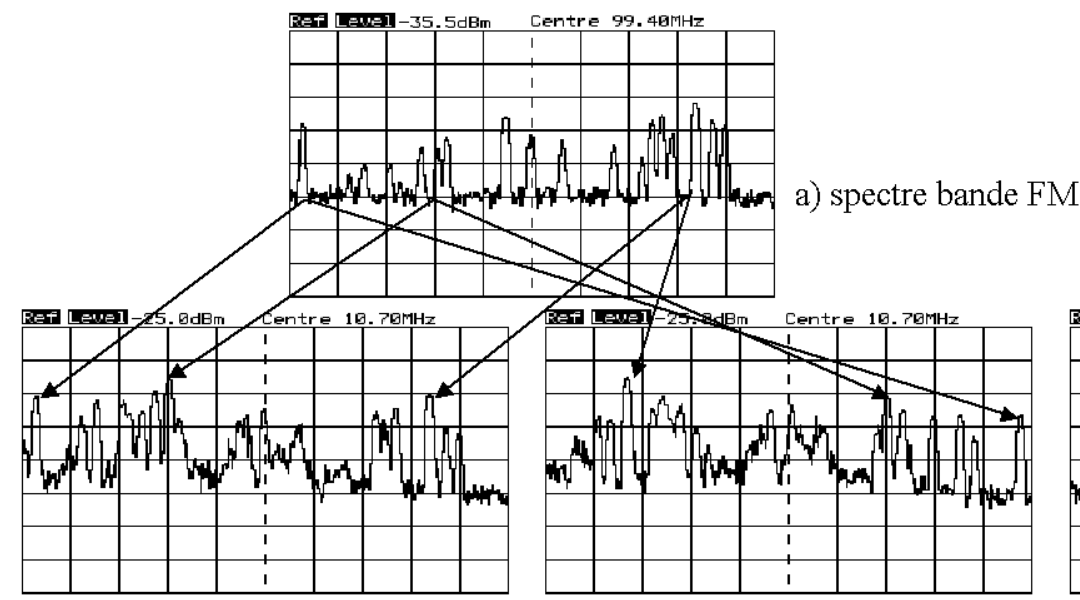

b) spectre à $\mathrm{FI}: \mathrm{f}_{\mathrm{OL}}=88,7 \mathrm{MHz}$ c) spectre à $\mathrm{FI}: \mathrm{f}_{\mathrm{OL}}=110,1 \mathrm{MHz}$

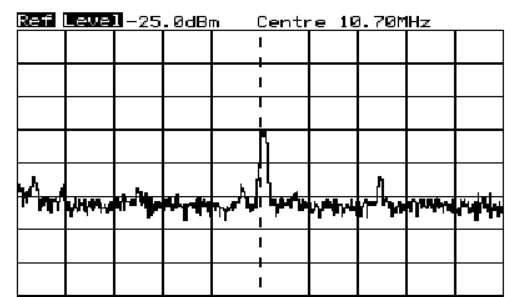

d) spectre après filtrage à FI

fig 2 : visualisation des spectres (graduations: verticale : $10 \mathrm{~dB} /$ div ; horizontale : $2 \mathrm{MHz} / \mathrm{div}$ ) : spectre de la bande FM (a); spectres en entrée du filtre FI, autour de FI, pour d'une réception infradyne (b) et pour d'une réception supradyne (c); spectre après filtrage à $F I\left(f_{O L}=110,1 \mathrm{MHz}\right)(d)$.

\subsection{Démodulateur en quadrature :}

Dans cette partie, le filtre céramique à $10,7 \mathrm{MHz}$ est déconnecté et on connecte à "l'entrée démodulateur" un synthétiseur de fréquence externe fonctionnant autour de $10,7 \mathrm{MHz}$ et modulable en fréquence (niveau de sortie : $0 \mathrm{dBm}$ ). On observe la tension sur la "sortie". Les étudiants doivent :
- Régler le condensateur variable du réseau déphaseur (synthétiseur de fréquence : 10,7 MHz, réglage du condensateur afin d'obtenir une tension de $2,25 \mathrm{~V}$, milieu de la caractéristique du démodulateur)

- Relever la caractéristique statique du démodulateur en quadrature en faisant varier la fréquence du synthétiseur (fig 3) 


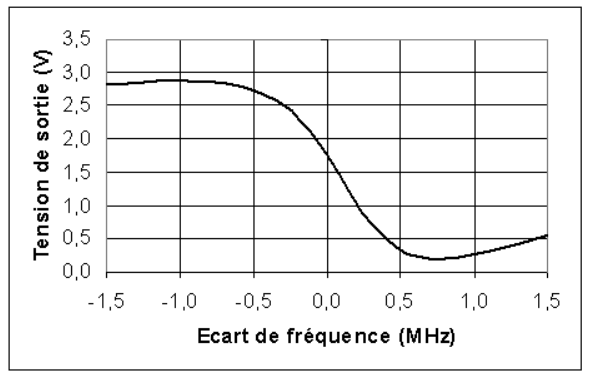

fig 3 : tension de sortie du démodulateur de fréquence en fonction de l'écart de fréquence par rapport à 10,7 MHz.

- Relever à l'oscilloscope, en XY, la caractéristique sortie du démodulateur - entrée du modulateur (le synthétiseur est réglé à une fréquence de $10,7 \mathrm{MHz}$ et est modulé en fréquence par un générateur basse fréquence externe, avec une excursion de fréquence de $75 \mathrm{kHz}$ ) (fig 4)

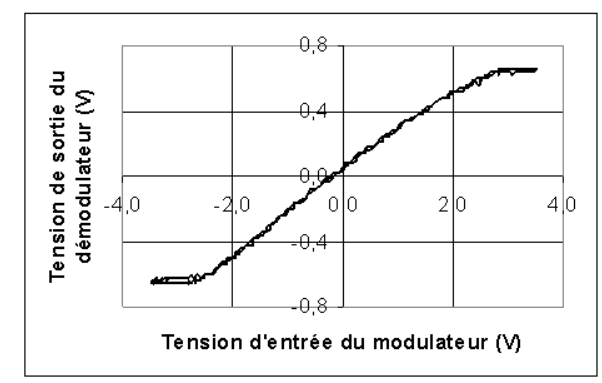

fig 4 : caractéristique sortie du démodulateur-entrée du modulateur pour une fréquence porteuse de 10,7 MHz.

- Relever le diagramme de Bode (gain et argument) de l'ensemble modulateur - démodulateur (entrée : signal modulant BF appliqué au synthétiseur, sortie : sortie du démodulateur) (fig 5)

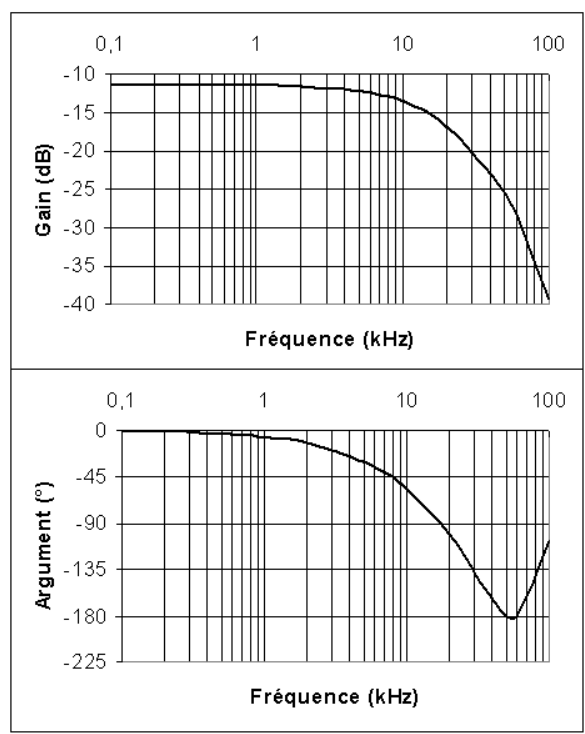

fig 5 : diagrammes de Bode de l'ensemble modulateurdémodulateur.

\subsection{Synthèse :}

A la fin du TP, les étudiants connecte le module haut-parleur et doivent effectuer les réglages nécessaires pour écouter une station donnée.

\section{ETUDE DU SYNTHETISEUR :}

L'étude et la programmation du synthétiseur de fréquence sont traitées en électronique et le mini projet se décompose en deux parties :

- Réalisation du circuit LC de l'oscillateur local et mise en œuvre du synthétiseur avec programmation par PC $(4 \mathrm{H})$

- Programmation du synthétiseur par l'intermédiaire du microcontrôleur $(8 \mathrm{H})$.

\subsection{Mise en ouvre de l'oscillateur local :}

Pendant la première phase, l'étudiant calcule et fabrique les différents composants constituant l'oscillateur local de récepteur. Il vérifie son bon fonctionnement.

Fabrication de la bobine de l'oscillateur local :

L'inductance L d'une bobine est fonction de la forme de la section de la bobine, de sa longueur $b$, de son diamètre $2 \mathrm{a}$ et du diamètre $\mathrm{d}$ du fil. Pour la fabrication des bobines à air ayant un facteur de qualité élevé, on peut admettre les règles suivantes:

1. $0,5<\mathrm{b} / 2 \mathrm{a}<2$

2. $2 \mathrm{a} / \mathrm{d}>5$

3. Pour les bobines de section circulaire, la meilleure approximation est la formule suivante :

$$
L=\mu_{0} n^{2} a \log \left(1+\pi \frac{a}{b}\right)+\frac{1}{2,3+1,6 a / b+0,44(a / b)^{2}}
$$

où $n$ est le nombre de spires, $\mu_{0}$ la permittivité de l'air, L est en Henry $(\mathrm{H})$, a et b en mètre $(\mathrm{m})$.

4. Pour une fréquence de $100 \mathrm{MHz}, \mathrm{L}$ de l'ordre de $0,1 \mu \mathrm{H}$.

L'étudiant doit :

- Calculer la valeur de $\mathrm{L}$ et de $\mathrm{C}\left(\mathrm{f}_{\mathrm{OL}}=110 \mathrm{MHz}\right)$

- Réaliser la bobine, implanter la bobine et le condensateur sur la maquette

- Placer le récepteur en boucle ouverte et régler, grâce au potentiomètre $\mathrm{P}, \mathrm{V} 23=2,5 \mathrm{~V}$

- Caler l'oscillateur à $110 \mathrm{MHz}$ en modifiant le profil de la bobine

- Relever la courbe $f_{O L}=f(V 23)$

- En déduire de cette courbe la plage de réception

- Brancher le module haut-parleur et régler le potentiomètre $\mathrm{P}$, pour permettre la réception de France Info.

\subsection{Mise en œuvre du synthétiseur :}

L'étudiant configure ensuite le synthétiseur via le PC (logiciel PLLGEN[5]) de manière à capter un émetteur local de la bande FM.

Fonctionnement du synthétiseur:

Le synthétiseur (fig 6) comporte deux diviseurs de fréquence par $\mathrm{R}$ et $\mathrm{N}$, un comparateur de phase 3 états, un oscillateur à quartz (MC145170), un VCO (MC3362) et un filtre de boucle. Les caractéristiques du comparateur 3 états sont données sur la fig 7.

Lorsque la boucle à verrouillage de phase est verrouillée, $f_{R}=f_{V}$ et la sortie $P_{\text {Dout }}$ est à l'état haute impédance : la tension de commande V23 du 
VCO correspond à la tension aux bornes du condensateur $\mathrm{C} 20$. Lorsque la fréquence $\mathrm{f}_{\mathrm{V}}$ est trop basse (fronts plus espacés), $\mathrm{P}_{\text {DOUT }}$ est plus souvent à $5 \mathrm{~V}$, ce qui charge le condensateur: la tension de commande du VCO augmente et $f_{\mathrm{V}}$ aussi. Un raisonnement similaire s'applique lorsque la fréquence $f_{V}$ est trop importante. Le réseau $\mathrm{R} 14, \mathrm{R} 4$ et $\mathrm{C} 20$, associé au comparateur de phase, joue aussi le rôle de filtre de boucle (stabilité du système).
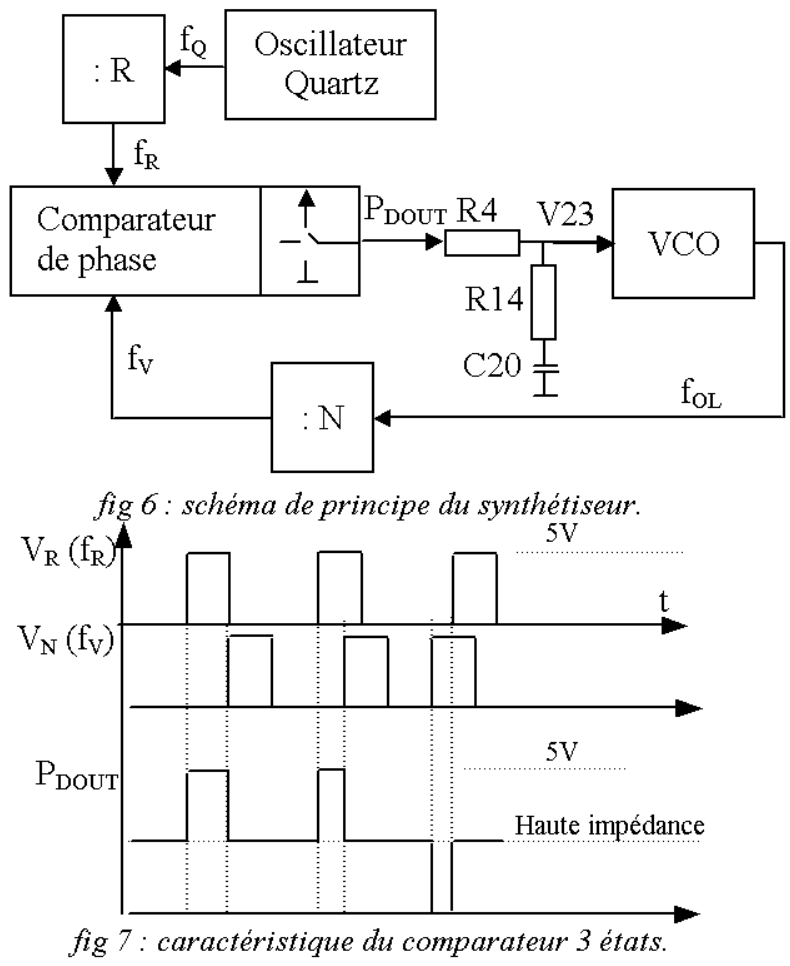

Calcul de $\mathrm{R}$ et $\mathrm{N}$ :

Le pas du synthétiseur $f_{R}$ est fixé par $f_{Q}$ et $R$ :

$$
\mathrm{R}=\frac{\mathrm{f}_{\mathrm{Q}}}{\mathrm{f}_{\mathrm{R}}}
$$

Si $\mathrm{f}_{\mathrm{FM}}$ est la fréquence de la station $\mathrm{FM}$ à recevoir, FI la fréquence intermédiaire du récepteur (ici, 10,7 $\mathrm{MHz}$ ), on a alors, pour une réception supradyne :

\section{Exemple:}

$$
\mathrm{N}=\frac{\mathrm{f}_{\mathrm{OL}}}{\mathrm{f}_{\mathrm{R}}}=\frac{\mathrm{f}_{\mathrm{FM}}+\mathrm{FI}}{\mathrm{f}_{\mathrm{R}}}
$$

$\mathrm{f}_{\mathrm{FM}}=100 \mathrm{MHz}, \mathrm{f}_{\mathrm{Q}}=10,240 \mathrm{MHz}, \mathrm{f}_{\mathrm{R}}=10 \mathrm{kHz}$, $\mathrm{FI}=10,7 \mathrm{MHz}$,

$$
\begin{aligned}
& \Rightarrow>R=1024 \\
& \Rightarrow>N=11070
\end{aligned}
$$

En ce qui concerne le synthétiseur, l'étudiant doit :

- Etudier la documentation constructeur, déterminer les valeurs du registre de contrôle $\mathrm{C}$ et des registres $\mathrm{R}$ et $\mathrm{N}$ permettant de recevoir France Info

- Connecter la maquette au $\mathrm{PC}$, placer le récepteur en boucle fermé, lancer le logiciel PLLGEN, charger la valeur de $\mathrm{C}, \mathrm{R}$ et $\mathrm{N}$

- Brancher le module haut-parleur, constater la réception de France Info, relever l'allure des signaux $f_{V}$ et $f_{R}$.

\section{- Conclure}

\subsection{Programmation du synthétiseur :}

Le but de la seconde phase du TP $(8 \mathrm{H})$ est de remplacer le $\mathrm{PC}$ par le module microcontrôleur. Durant cette phase, on demande à l'étudiant de :

- Elaborer les différents sous programmes permettant la configuration des trois registres

- Ecrire le programme en langage évolué (basic ou C) correspondant à l'ordinogramme de la fig 8 .

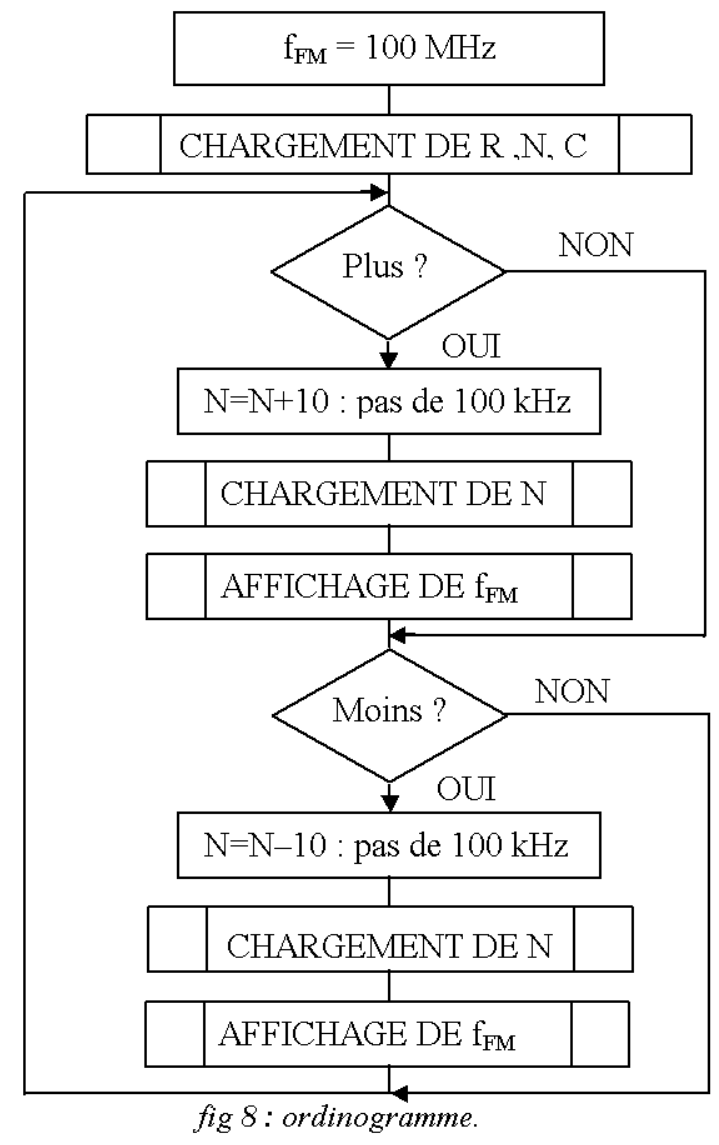

\section{CONCLUSION :}

Cette maquette a permis, grâce à son faible coût ( 60 euros), de faire manipuler les étudiants sur un récepteur hétérodyne. Ces derniers, dont certains sont inscrits au radio club du lycée (responsable : J. SALLE) ont ainsi pu modifier des radiotéléphones $\mathrm{BOSCH}$ pour en faire des récepteurs $\mathrm{FM}$ à double changement de fréquence autour de $144 \mathrm{MHz}$.

\section{Bibliographie :}

[1] BTS Électronique 1990 - Physique Appliquée.

[2] Documentation technique MC3362 (Motorola) www.qls.net/dloaz/datasheets

[3] Documentation MC145170 www.motorola.com

[4] Documentation 89S53 www.atmel.com

[5] Logiciel PLLGEN www.qls.net

[6] R. Werlé (2002), Une initiative exemplaire à Sète, Megahertz magazine, $n^{\circ} 236$, p 16-18 